\title{
A PARTITION PROPERTY CHARACTERIZING CARDINALS HYPERINACCESSIBLE OF FINITE TYPE
}

BY

\author{
JAMES H. SCHMERL
}

ABSTRACT. Let $\mathrm{P}(n, \alpha)$ be the class of infinite cardinals which have the following property: Suppose for each $\nu<\kappa$ that $C_{\nu}$ is a partition of $[\kappa]^{n}$ and card $\left(C_{\nu}\right)<\kappa$; then there is $X \subset \kappa$ of length $\alpha$ such that for each $\nu<\kappa$, the set $X-(\nu+1)$ is $C_{\nu}$-homogeneous. In this paper the classes $\mathbf{P}(n, \alpha)$ are studied and a nearly complete characterization of them is given. A principal result is that $\mathrm{P}(n+2, n+5)$ is the class of cardinals which are hyperinaccessible of type n.

A partition property which differs from usual ones in that many partitions are considered simultaneously is defined and investigated in this paper. This property is interesting because it leads to an elementary characterization of the class of cardinals which are hyperinaccessible of a given finite type. The motivation for such a characterization comes from a problem in model theory. This problem is satisfactorily solved (as announced in [4] and [5]) using some of the combinatorial results of this paper. These results lead naturally to a combinatorial problem which seems to be of sufficient independent interest so as to warrant further investigation. We give here an almost complete solution of this combinatorial problem; the final step for a complete solution seems elusive.

This paper is a reworked version of Chapter 7 of my $\mathrm{Ph}$. D. thesis [6] written under the supervision of Profess or Robert L. Vaught. The other parts of my thesis, which consist of the model-theoretic applications of the results included here as well as generalizations of these results, will appear elsewhere.

1. The basic concepts. An ordinal number is the set of its predecessors. Ordinals are denoted by the Greek letters $a, \beta, \gamma, \nu, \xi$. Cardinal numbers are identified with initial ordinals and are denoted by $\kappa, \lambda$, $\mu$, where $\kappa$ is always an infinite cardinal. The symbols $n, m, i$ always denote finite ordinals.

A cardinal $\kappa$ is a strong limit cardinal iff $2^{\lambda}<\kappa$ whenever $\lambda<\kappa$. An inaccessible cardinal is a regular, strong limit cardinal. We need the concept of a

Received by the editors June 17, 1971.

AMS (MOS) subject classifications (1970). Primary 02X35, 04A10; Secondary 02H05, $02 \mathrm{H} 13$.

Key words and phrases. Partition, inaccessible cardinal, hyperinaccessible cardinal. 
hyperinaccessible cardinal of type $n$ (as given by Lévy [2]); we adopt the terser term of $n$-inaccessible.

1.1. Definition. We define $n$-inaccessible by induction on $n$ so that $\kappa$ is 0 -inaccessible iff it is inaccessible; and $\kappa$ is $(n+1)$-inaccessible iff every closed, cofinal subset of $\kappa$ contains an $n$-inaccessible.

We make the natural, albeit unusual, convention that $\omega$ is $n$-inaccessible, although we generally dismiss this case without mention.

A partition $C$ of the set $X$ is a collection of pairwise disjoint sets, the union of which is $X$. Two elements in the same set of $C$ are called $C$-equivalent. The set of subsets of $X$ of cardinality $n$ is denoted by $[X]^{n}$. We tacitly make the natural identification of $X$ and $[X]^{1}$. If $C$ is a partition of $[X]^{n}$, then $Y \subset X$ is $C$ homogeneous iff every two elements of $[Y]^{n}$ are $C$ equivalent. We denote by $f[X]$ the set $\{f(x): x \in X\}$.

The following definitions introduce concepts fundamental to this paper.

1.2. Definition. (i) $C$ is an f-partition system of $[a]^{n}$ iff $f$ is a cardinal-valued function with domain including $a$ such that for each $\nu<a, C_{\nu}$ is a partition of $[a]^{n}$ and $\operatorname{card}\left(C_{\nu}\right) \leq f(\nu)$.

(ii) $C$ is a partition system of $[\alpha]^{n}$ iff for some $f: a \rightarrow a, C$ is an f-partition system of $[a]^{n}$.

(iii) If $C$ is an $f$-partition system of $[\alpha]^{n}$ then $X \subset \alpha$ is $C$-bomogeneous iff for each $\nu \in X$, the set $X-(\nu+1)$ is $C_{\nu}$-homogeneous.

(iv) $\mathbf{P}(n, \alpha)$ is the class of all $\kappa$ such that for any partition system $C$ of $[k]^{n}$ there is a $C$-homogeneous set of length $a$.

The question we investigate is this: Which cardinals are in $\mathbf{P}(n, a)$ ? In $\$ 2$ we give a complete solution for accessibles. In $\$ 3$ we give sufficient conditions on an inaccessible $\kappa$ in order that $\kappa \in \mathrm{P}(n, a)$; and in $\$ 4$ we give necessary conditions on an inaccessible $\kappa$ in order that $\kappa \in \mathbf{P}(n, a)$. In $\$ 5$ we state an open problem.

1.3. Proposition. If $\alpha<\beta$ and $\kappa \in \mathbf{P}(n, \beta)$, then $\kappa \in \mathbf{P}(n, \alpha)$.

1.4. Proposition. If $\kappa \in \mathrm{P}(n, \alpha)$, then $\alpha \leq \kappa$.

1.5. Proposition. $\kappa \in \mathrm{P}(1,3)$ and $\kappa \in \mathrm{P}(n, n+1)$.

1.6. Theorem. $\omega \in \mathbf{P}(n, \omega)$.

Proof. This is an easy consequence of Ramsey's theorem [3]. Define $n_{i}$ and $X_{i}$ inductively as follows. Let $X_{0}=\omega, n_{i}=\min \left(X_{i}\right)$, and let $X_{i+1}$ be an infinite $C_{n_{i}}$-homogeneous subset of $X_{i}-\left\{n_{i}\right\}$. Clearly, then, $\left\{n_{i}: i<\omega\right\}$ is an infinite $C$ homogeneous set.

One proof of Ramsey's theorem is by induction on $n$ and makes use of a tree 
construction. We could also have proved the previous theorem by induction on $n$ and by use of a tree, thus eliminating the appeal to Ramsey's theorem. Since there are several occasions in this paper to use such trees, we here make their definition explicit.

Recall that $T=(A,<)$ is a tree iff $T$ is a partially-ordered set and the set of predecessors of any element is well ordered by $<$. Also $T_{\nu}$ is the set of elements of rank $\nu$, that is, those elements whose set of predecessors has length $\nu$. The tree $T$ is a $\kappa$-tree iff $0<\operatorname{card}\left(T_{\nu}\right)<\kappa$ for each $\nu<\kappa$ and $T_{\kappa}=\varnothing$.

Let $C$ be a partition system of $[a]^{n+1}$. We define inductively the tree $(\alpha$, $\unlhd$ ) which we call the associated tree. If $\beta, \gamma<\alpha$, then $\beta \unlhd \gamma$ iff $\beta \leq \gamma$ and whenever $A \in[\{\xi<\alpha: \xi \triangleleft \beta\}]^{n}$ and $\nu \triangleleft \beta$, then $A \cup\{\beta\}$ and $A \cup\{\gamma\}$ are $C_{\nu}$-equivalent. It is easily verified that $(\alpha, \unlhd)$ is indeed a tree. We often make use of the following fact: if $C$ is a partition system of $[\kappa]^{n+1}$ where $\kappa$ is inaccessible, then the associated tree is a $k$-tree.

2. The accessible case. In this section a complete characterization of those accessible cardinals in $\mathbf{P}(n, \alpha)$ is given.

2.1. Theorem. If $\kappa \in \mathbf{P}(n+1, n+4)$ then $\kappa$ is regular.

Proof. Suppose $\kappa$ is singular. There is a set $Y$ cofinal in $\kappa$ such that $\operatorname{card}(Y)<\kappa$. Let $g: \kappa \rightarrow Y$ be defined by $g(\nu)=\min (Y-(\nu+1))$. Let $C$ be a partition system of $[\kappa]^{n+1}$ such that whenever $\nu<\kappa$ and $A, B \in[\kappa]^{n+1}$, then $A$ and $B$ are $C_{\nu}$ equivalent iff one of the following holds:

(i) $g(\nu) \leq \min (A)$ and $A=B$.

(ii) $\min (A)<g(\nu)$ and $g(\nu) \cap A=g(\nu) \cap B$.

Clearly, $C$ is a partition system of $[k]^{n+1}$ and there is no $C$-homogeneous set of length $n+4$.

\subsection{Theorem. $\kappa \in \mathbf{P}(n+2, n+4)$ iff $\kappa$ is a strong limit cardinal.}

Proof. Suppose $\alpha$ is not a strong limit cardinal, so that $\lambda<\kappa \leq 2^{\lambda}$ for some $\lambda$. As is well known, there is a partition $D$ of $[\kappa]^{n+2}$ such that card $(D) \leq \lambda$ and such that no $D$-homogeneous set has length $n+3$. (For example, let $\left\langle a_{\nu}: \nu\langle\kappa\rangle\right.$ be a sequence of distinct subsets of $\lambda$. If $\nu_{0}<\ldots<\nu_{n+1}<\kappa$ and $\xi_{0}<\ldots<\xi_{n+1}$ $<\kappa$, then let $\left\{\nu_{0}, \cdots, \nu_{n+1}\right\}$ and $\left\{\xi_{0}, \cdots, \xi_{n+1}\right\}$ be Dequivalent iff $\min \left(\left(a_{\nu_{0}}-a_{\nu_{1}}\right) \cup\left(a_{\nu_{1}}-a_{\nu_{0}}\right)\right)=\min \left(\left(a_{\xi_{0}}-a_{\xi_{1}}\right) \cup\left(a_{\xi_{1}}-a_{\xi_{0}}\right)\right)$.) Consider the partition system $C$ of $[\kappa]^{n+2}$ where $C_{\nu}=D$ for each $\nu<\kappa$. Then there is no $C$-homogeneous set of length $n+4$.

Now suppose $\kappa$ is a strong limit cardinal, and let $C$ be a partition system of $[\kappa]^{n+2}$. By the well-known Erdös-Rado theorem (see [1, Theorem 39]), there is a $C_{0}$-homogeneous set $X \subset K-\{0\}$ of length $n+3$. Then $X \cup\{0\}$ is $C$-homogeneous and has length $n+4$. 
2.3. Corollary. If $\kappa \in \mathrm{P}(n+2, n+5)$, then $\kappa$ is inaccessible.

2.4. Theorem. Let $\kappa$ be regular and accessible, and let $\lambda$ be the smallest cardinal sucb that $\kappa \leq \mu^{\lambda}$ for some $\mu<\kappa$. Then $\kappa \in \mathrm{P}(1, a)$ iff $a \leq \lambda+1$.

Proof. We first show that $\kappa \in \mathrm{P}(1, \lambda+1)$. Let $C$ be a partition system of $\kappa$, and let $T=(\kappa, \unlhd)$ be the associated tree. It is clear by induction that $\operatorname{card}\left(T_{\nu}\right)$ $<\kappa$ for each $\nu<\lambda$. Hence $T_{\lambda} \neq \varnothing$. Choose some $\beta \in T_{\lambda}$; then it is easily shown that $\{\gamma: \gamma \unlhd \beta\}$ has length $\lambda+1$ and is $C$-homogeneous.

Now we show that $\kappa \notin \mathrm{P}(1, \lambda+2)$. Let $\mu<\kappa$ be such that $\kappa \leq \mu^{\lambda}$. Let $\left\langle_{8}\right.$ : $a<\kappa)$ be a sequence of distinct elements of $\lambda_{\mu}$. For each $\nu<\kappa, \alpha<\lambda$ and $\beta<\mu$, let $E(\nu, \alpha, \beta)=\left\{\eta<\kappa: \nu<\eta, g_{\eta}(\alpha)=\beta\right.$ and $\alpha$ is the least ordinal for which $\left.g_{\nu}(\alpha) \neq g_{\eta}(\alpha)\right\}$. For each $\nu<\kappa$ let $C_{\nu}=\{E(\nu, \alpha, \beta): \alpha<\lambda$ and $\beta<\mu\} \cup$ $\{\{\nu+1\}\}$. Clearly, $C$ is a partition system of $\kappa$.

We now show that there is no $C$-homogeneous set of length $\lambda+2$. Suppose that $X=\left\{\nu_{\xi}: \xi<\lambda+2\right\}$ were such a set where $\nu_{0}<\nu_{1}<\ldots$. For each $\xi<\lambda+$ 2 there are $\alpha_{\xi}<\lambda$ and $\beta_{\xi}<\mu$ such that $X-\left(\nu_{\xi}+1\right) \subset E\left(\nu_{\xi}, a_{\xi}, \beta_{\xi}\right)$.

Now suppose $\xi<\eta<\lambda+1$. We show that $\alpha_{\xi}<\alpha_{\eta}$. Whenever $\alpha<a_{\xi}$, then $\boldsymbol{g}_{\nu_{\xi}}(a)=\boldsymbol{g}_{\nu_{\eta}}(a)=\boldsymbol{g}_{\nu_{\lambda+1}}(a)$. But also, $\boldsymbol{g}_{\nu_{\eta}}\left(a_{\xi}\right)=\boldsymbol{g}_{\nu_{\lambda+1}}\left(a_{\xi}\right)=\beta_{\xi}$. Hence $a_{\xi}<$ $a_{\eta}$. Thus we have shown that $\left\langle a_{\xi}: \xi\langle\lambda+1\rangle\right.$ is a strictly increasing sequence of ordinals $<\lambda$. This is a contradiction.

3. Sufficient conditions for the inaccessible case. The proofs of the following two theorems are the main goals of this section.

3.1. Theorem. If $\kappa$ is n-inaccessible and $a<\kappa$, then $\kappa \in \mathbf{P}(n+1, a)$.

3.2. Theorem. If $\kappa$ is n-inaccessible, then $\kappa \in \mathbf{P}(n+2, m)$.

Each of these theorems is proved by an induction on $n$. The next lemma captures the essence of the induction steps.

3.3. Lemma. Let $\kappa$ be inaccessible sucb that for eacb closed, cofinal subset $X$ of $\kappa, X \cap \mathbf{P}(n, a) \neq \varnothing$. Then $\kappa \in \mathbf{P}(n+1, a+1)$.

Proof . Let $C$ be a partition system of $[\kappa]^{n+1}$ and let $T=(\kappa, \unlhd)$ be the associated tree. Let $f: \kappa \rightarrow \kappa$ be such that if $\nu<\kappa$ and $\xi \in T_{\nu}$, then $\operatorname{card}\left(C_{\xi}\right)<f(\nu)$. The set $X=\{\xi<\kappa: f[\xi] \subset \xi\}$ is a closed, cofinal subset of $\kappa$; hence, there is $\lambda \epsilon$ $X \cap P(n, a)$. Since $T_{\lambda} \neq \varnothing$ we can choose $\gamma \in T_{\lambda}$. For each $\xi \leq \lambda$ let $g(\xi) \in T_{\xi}$ be such that $g(\xi) \unlhd \gamma$.

Now let $D$ be a partition system of $[\lambda]^{n}$ such that whenever $\nu<\lambda$ and $A, B$ $\epsilon[\lambda]^{n}$, then $A$ and $B$ are $D_{\nu}$ equivalent iff $g[A \cup\{\lambda\}]$ and $g[B \cup\{\lambda\}]$ are $C_{g(\nu)^{-}}$ equivalent. If $\nu<\lambda$ then $\operatorname{card}\left(D_{\nu}\right) \leq \operatorname{card}\left(C_{g(\nu)}\right)<f(\nu)<\lambda$, so that $D$ is a partition system of $[\lambda]^{n}$. Hence, there is a $D$-homogeneous set $Z$ of length $a$. Let 
$Y=g[Z \cup\{\lambda\}]$. We show that $Y$ is $C$-homogeneous.

Let $\nu \in Y$ and $A, B \in[Y-(\nu+1)]^{n+1}$. Let $\alpha=\max (A), \beta=\max (B), A^{\prime}=$ $A-\{a\}$ and $B^{\prime}=B-\{\beta\}$. Then $A$ and $A^{\prime} \cup\{g(\lambda)\}$ are $C_{\nu}$-equivalent due to the manner of construction of the associated tree $T$. Similarly, $B$ and $B^{\prime} \cup\{g(\lambda)\}$ are $C_{\nu}$ equivalent. Furthermore, $A^{\prime} \cup\{g(\lambda)\}$ and $B^{\prime} \cup\{g(\lambda)\}$ are $C_{\nu}$ equivalent

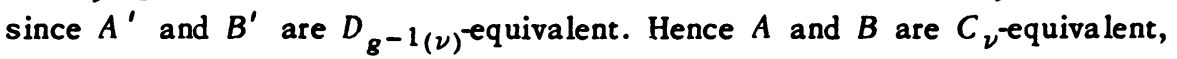
so that $Y$ is $C$-homogene ous.

Proof of 3.1. Suppose that $n=0$ and that $C$ is a partition system of $\kappa$. Let $T=(\kappa, \unlhd)$ be the associated tree. Since $T_{a} \neq \varnothing$ we can choose $\gamma \in T_{\alpha}$. Then $\{\nu: \nu \unlhd \gamma\}$ is a $C$-homogeneous set of length $a$. Use induction with Lemma 3.3 to complete the proof.

The proof of Theorem 3.2 is more difficult in that the proof of the basis step of the induction is more involved.

3.4. Definition. Let $f: a \rightarrow a$. Then we define $K_{n}(f)$ inductively by $K_{0}(f)=$ $\{\kappa \leq a: f[\kappa] \subset \kappa\}$ and $K_{n+1}(f)=\left\{\kappa \in K_{n}(f): \operatorname{card}\left(\kappa \cap K_{n}(f)\right)=\kappa\right\}$.

3.5. Lemma. Let $f: \kappa \rightarrow \kappa$ be sucb tbat $\kappa \in K_{n}(f)$, and let $C$ be a partition of $\alpha$ such that card $(C)<c f(\kappa)$. Then there exists a strictly increasing function $b: \kappa \rightarrow \kappa$ sucb that $b[\kappa] \in C$ and $\kappa \in K_{n}(b \circ f)$.

Proof. We use induction on $n$. For the case $n=0$ the lemma is trivial. Assume that is it true for $n=m$; we show that it is true for $n=m+1$.

Let $f: \kappa \rightarrow \kappa$ be such that $\kappa \in K_{m+1}(f)$, and let $C$ be a partition of $\kappa$ such that card $(C)<c f(\kappa)$. Let $X=\left\{\lambda \in K_{m}(f)\right.$ : $\left.\operatorname{card}(C)<c f(\lambda)\right\}$. We show that card $(X)$ $=\kappa \cdot K_{m}(f) \cap \kappa$ is closed in $\kappa$ and has cardinality $\kappa$ since $\kappa \in K_{m+1}(f)$. Let $g: \kappa \rightarrow K_{m}(f)$ be a continuous, strictly increasing function. Then it is clear that $\left\{g\left(\lambda^{+}\right)\right.$: card $\left.(C) \leq \lambda<\kappa\right\}$ has cardinality $\kappa$ and that whenever card $(C) \leq \lambda<\kappa$, then $c f\left(g\left(\lambda^{+}\right)\right)=c f\left(\lambda^{+}\right)=\lambda^{+}>\operatorname{card}(C)$.

We now use the induction hypothesis. For each $\lambda \in X$ let $C_{\lambda}=\{E \cap \lambda: E \in C\}$ be a partition of $\lambda$. Then there is a strictly increasing function $b_{\lambda}: \lambda \rightarrow \lambda$ such that $b_{\lambda}[\lambda] \in C_{\lambda}$ and $\lambda \in K_{m}\left(b_{\lambda} \circ f\right)$. Since card $(X)=\kappa$ and card $(C)<c f(\kappa)$ there are $E \in C$ and $Y \subset X$ such that $\operatorname{card}(Y)=\kappa$ and $b_{\lambda}[\lambda]=E \cap \lambda$ whenever $\lambda \epsilon Y$. Then card $(E)=\kappa$. Now let $b: \kappa \rightarrow \kappa$ be a strictly increasing function such that $b[\lambda]=E$. (Notice that $b(\nu)=b_{\lambda}(\nu)$ whenever $\nu<\lambda \in Y$.) If $\lambda \in Y$ then $\lambda \epsilon$ $K_{m}\left(b_{\lambda} \circ f\right) \subset K_{m}(b \circ f)$; hence $Y \subset K_{m}(b \circ f)$. Therefore, since card $(Y)=\kappa$, it follows that $\kappa \in K_{m+1}(b \circ f)$.

3.6. Lemma. Let $f: \kappa \rightarrow \kappa$ be sucb that $\kappa \in K_{n}(f)$, and let $C$ be an f-partition system of $\kappa$. Then there is a $C$-bomogeneous set of lengtb $n+3$.

Proof. We use induction on $n$. For $n=0$, the lemma follows from Proposition 1.5. Suppose the lemma is true for $n=m$; we show it is true for $n=m+1$. 
Let $f: \kappa \rightarrow \kappa$ be such that $\kappa \in K_{m+1}(f)$, and let $C$ be an $f$-partition system of $\kappa$. Since $\left.\kappa \in K_{m}+1\right\}$, there is $\lambda \in K_{m}(f)$ such that $f(0)<c f(\lambda)$. By Lemma 3.5 there is a strictly increasing $b: \lambda \rightarrow \lambda$ and $E \in C_{0}$ such that $b[\lambda]=E \cap \lambda$ and $\lambda \in K_{m}(b \circ f)$.

Now let $D$ be a partition system of $\lambda$ defined as follows: if $\nu, \nu_{0}, \nu_{1}<\lambda$, then $\nu_{0}$ and $\nu_{1}$ are $D_{\nu}$-equivalent iff $b\left(1+\nu_{0}\right)$ and $b\left(1+\nu_{1}\right)$ are $C_{b(1+\nu)}$ equivalent. Thus, whenever $\nu<\lambda$, then card $\left(D_{\nu}\right) \leq \operatorname{card}\left(C_{b(1+\nu)}\right) \leq f(b(1+\nu))<\lambda$. Us ing the induction hypothesis, there is a $D$-homogeneous set $Y \subset \lambda$ of length $m+3$. It is clear that $\{0\} \cup\{b(1+\nu): \nu \in Y\}$ is $C$-homogeneous and has length $m+4$.

Proof of 3.2. Suppose that $n=0$ and that $C$ is an $/$-partition system of $[k]^{2}$, where $f: \kappa \rightarrow \kappa$. Let $T=(\kappa, \unlhd)$ be the associated tree. Let $b: \kappa \rightarrow \kappa$ be such that if $\xi<\kappa$ and $\nu \in T_{\xi}$, then $f(\nu)<b(\xi)$. Since $\kappa$ is inaccessible, it is clear that $\kappa \cap K_{m}(b) \neq \varnothing$. Choose $\lambda \in \kappa \cap K_{m}(b)$. Since $T_{\lambda} \neq \varnothing$ we can choose $\gamma \in$ $T_{\lambda}$. For each $\xi \leq \lambda$ let $g(\xi) \in T_{\xi}$ be such that $g(\xi) \unlhd \gamma$.

Now let $D$ be a partition system of $\lambda$ such that whenever $\nu, \nu_{0}, \nu_{1}<\lambda$, then $\nu_{0}$ and $\nu_{1}$ are $D$-equivalent iff $\left\{g\left(\nu_{0}\right), g(\lambda)\right\}$ and $\left\{g\left(\nu_{1}\right), g(\lambda)\right\}$ are $C_{g(\nu)}$ equivalent. Then card $\left(D_{\nu}\right) \leq \operatorname{card}\left(C_{g(\nu)}\right)<b(\nu)$. By Lemma 3.6 there is a $D$-homogeneous set $Z$ of length $m+3$. Let $Y=g[Z \cup\{\lambda\}]$. As in the proof of Lemma 3.3, the set $Y$ is $C$-homogeneous.

Use the induction with Lemma 3.3 to complete the proof.

We remark that the condition $a<\kappa$ in the hypothesis of Theorem 3.1 can be dropped iff $\alpha$ is weakly compact. (Here it is useful to define $\alpha$ as weakly compact iff $\kappa$ is inaccessible and for every tree $T$, if $0<\operatorname{card}\left(T_{\nu}\right)<\kappa$ whenever $\nu<$ $\kappa$, then $T$ has a branch of length $\kappa$.) Thus, we have the following theorem.

\subsection{Theorem. $\kappa \in \mathbf{P}(n+1, \kappa)$ iff $\kappa$ is weakly compact.}

We do not give a proof but do note that to prove the implication from right to left one uses Lemma 3.3, and to prove the implication from left to right one uses a construction similar to that in Theorem 2.4.

4. Necessary conditions for the inaccessible case. The proofs of the following two theorems are the main goals of this section.

4.1. Theorem. If $\kappa \in \mathbf{P}(n+2, \omega+n+1)$, then $\kappa$ is $(n+1)$-inaccessible.

4.2. Theorem. If $\kappa \in \mathbf{P}(n+2, n+5)$, then $\kappa$ is $n$-inaccessible.

Each of these theorems is proved by an induction on $n$. The following definitions and the two succeeding lemmas are used in the induction steps.

4.3. Definition. (i) A function $f: \kappa \rightarrow \kappa$ is $m$-normal iff $f$ is a continuous, strictly increasing function such that whenever $\nu<\kappa$, then $f(\nu)$ is a strong limit cardinal which is not $m$-inaccessible. 
(ii) $S_{1}(m, n, \gamma)$ iff for every inaccessible $\kappa$ and every $m$-normal function $f: \kappa \rightarrow \kappa$, there is an $f$-partition system $C$ of $[\kappa]^{n}$ such that each $C$-homogeneous set has length $<\gamma$.

(iii) $S_{2}(m, n, \gamma)$ iff for every inaccessible $\kappa$, for every $a<\kappa$, and for every $m$-normal function $f: \kappa \rightarrow \kappa$, there is an $f$-partition system $C(\alpha)$ of $[\alpha]^{n}$ such that each $C(a)$-homogeneous set has length $<\gamma$.

4.4. Proposition. If $\kappa$ is inaccessible, then there is an n-normal $f: \kappa \rightarrow \kappa$ iff $K$ is not $(n+1)$-inaccessible.

4.5. Lemma. If $S_{2}(m, n, \gamma)$ then $S_{1}(m, n+1, \gamma+1)$.

Proof. Let $\kappa$ be inaccessible and let $f: \kappa \rightarrow \kappa$ be an m-normal function. For each $a<\kappa$, let $C(\alpha)$ be an /-partition system of $[a]^{n}$ such that each $C(a)$. homogeneous set has length $\left\langle\gamma\right.$. Let $H_{\nu, a}:[\alpha]^{n} \rightarrow f(\nu)$ be such that whenever $A, B \in[a]^{n}$, then $A$ and $B$ are $C_{\nu}(a)$ equivalent iff $H_{\nu, a}(A)=H_{\nu, a}(B)$. Then let $D_{\nu}^{\xi}=\left\{A \in[\kappa]^{n+1}: H_{\nu, a}(A-\{a\})=\xi\right.$ where $\left.a=\max (A)\right\}$, and let $D_{\nu}=\left\{D_{\nu}^{\xi}: \xi<\right.$ $f(\nu)\}$. Clearly $D$ is an $f$-partition system of $[k]^{n+1}$. If $X$ is a $D$-homogeneous set of length $\gamma+1$ and $a=\max (X)$, then $X-\{a\}$ is $C(a)$-homogeneous. Thus every $D$-homogeneous set has length $<\gamma+1$.

4.6. Lemma. Suppose that $2 \leq n, n+3 \leq \gamma$ and for each $i<m, S_{1}(i, n, \gamma)$. Then $S_{2}(m, n, \gamma)$.

Proof. Assume the hypothesis of the lemma, let $\kappa$ be inaccessible and let $f: \kappa \rightarrow \kappa$ be an $m$-normal function. By Proposition $4.4 \kappa$ is not $(m+1)$-inaccessible.

We show by induction on ordinals that for each $\alpha<\kappa$ there is an $f$-partition system $C(\alpha)$ of $[\alpha]^{n}$ such that every $C(\alpha)$-homogeneous set has length $<\gamma$. Let $\alpha<\kappa$ and assume that for each $\beta<\alpha$ such a $C(\beta)$ exists; we prove such a $C(\alpha)$ exists.

Case 1. $\alpha \leq \omega$. This case is trivial since $f(\nu) \geq \omega \geq \alpha$ whenever $\nu<\kappa$.

Case 2. $\alpha>\omega$ and $\alpha \notin f[\kappa]$. Then there is $\beta$ such that $\beta<\alpha<f(\beta)$. Let $C(\alpha)$ be the partition system of $[\alpha]^{n}$ such that whenever $\nu<\alpha$ and $A, B \in[a]^{n}$, then $A$ and $B$ are $C_{\nu}(\alpha)$-equivalent iff one of the following holds:

(i) $\nu<\beta$ and $\operatorname{card}(A \cap \beta)=\operatorname{card}(B \cap \beta)<n$.

(ii) $\nu<\beta$, and $A$ and $B$ are $C_{\nu}(\beta)$-equivalent.

(iii) $\beta \leq \nu$ and $A=B$.

It is clear that $C(\alpha)$ is an $f$-partition system of $[\alpha]^{n}$.

Suppose that $X$ is $C(a)$-homogeneous. Let $\nu_{0}=\min (X)$ and $\nu_{1}=\min \left(X-\left\{\nu_{0}\right\}\right)$. The induction hypothesis and (ii) jointly imply that $X-\beta \neq \varnothing$. But then (i) implies that $\beta \leq \nu_{1}$, and then (iii) implies that card $(X-\beta) \leq n+1$. Hence, card $(X)$ $\leq n+2<\gamma$. 
Case 3. $a>\omega$ and $a \in f[\kappa]$. Then either (i) $\alpha$ is singular, or (ii) $\alpha$ is inaccessible but not $m$-inaccessible (in which case $m \geq 1$ ). Let $\lambda=c f(\alpha)$. Then let $g: \lambda \rightarrow \alpha$ and $D$ be a partition sytem of $[\lambda]^{n}$ as follows:

(i) If $\alpha$ is singular, let $g: \lambda \rightarrow \alpha$ be a continuous, strictly increasing function cofinal in $a$, and let $D=C(\lambda)$.

(ii) If $\alpha$ is inaccessible, let $g: \alpha \rightarrow \alpha$ be an $(m-1)$-normal function (by Proposition 4.4), and let $D$ be a $g$-partition system of $[a]^{n}$ such that any $D$-homogeneous set has length $<\gamma$.

Now let $b: \alpha \rightarrow \lambda$ be such that $b(\nu)=\sup \{\beta<\lambda: g(\beta) \leq \nu\}$. Let $H_{\nu, \beta}:[g(\beta)]^{n}$ $\rightarrow f(\nu)$ be such that whenever $A, B \in[g(\beta)]^{n}$, then $A$ and $B$ are $C_{\nu}(g(\beta))$-equivalent iff $H_{\nu, \beta}(A)=H_{\nu, \beta}(B)$.

We are now ready to define the $f$-partition system $C(\alpha)$. Let $\nu<a$, and let $A=\left\{a_{0}, \cdots, a_{n-1}\right\}$ and $B=\left\{b_{0}, \cdots, b_{n-1}\right\}$ where $a_{0}<\cdots<a_{n-1}<a$ and $b_{0}<\cdots<b_{n-1}<a$. Then $A$ and $B$ are $C_{\nu}(a)$-equivalent iff one of the following conditions is satisfied:

(E1) $b(\nu)<b\left(a_{0}\right)<\cdots<b\left(a_{n-1}\right) ; b(\nu)<b\left(b_{0}\right)<\cdots<b\left(b_{n-1}\right) ; b[A]$ and $b[B]$ are $D_{b(\nu)}$ equivalent.

(E2) $\nu<a_{0}, b_{0} ; b(\nu)=b\left(a_{0}\right)<\cdots<b\left(a_{n-1}\right) ; b(\nu)=b\left(b_{0}\right)<\cdots<b\left(b_{n-1}\right)$.

(E3) $\nu<a_{0}, b_{0} ; b\left(a_{0}\right)=\cdots=b\left(a_{n-1}\right) ; b\left(b_{0}\right)=\cdots=b\left(b_{n-1}\right) ; H_{\nu, b\left(a_{0}\right)+1}(A)=$ $H_{\nu, b\left(b_{0}\right)+1}(B)$.

(E4) $\nu<a_{0}, b_{0} ; 2 \leq \operatorname{card}(b[A])<n$; whenever $i<j<n$, then $b\left(a_{i}\right)=b\left(a_{j}\right)$ iff $b\left(b_{i}\right)=b\left(b_{j}\right)$.

(ES) $a_{0} \leq \nu$ and $A \cap(\nu+1)=B \cap(\nu+1)$.

It is easily checked that (E1)-(ES) constitute a proper definition in order that $C(a)$ be an $f$-partition system of $[a]^{n}$.

It remains to check that whenever $X$ is $C(\alpha)$-homogeneous, then $X$ has length $<\gamma$. Suppose to the contrary that $X=\left\{\nu_{\xi}: \xi<\gamma\right\}$ is $C(\alpha)$-homogeneous, where $\nu_{0}<\nu_{1}<\cdots$. Whenever $\xi+n-1<\gamma$ let $A_{\xi}=\left\{\nu_{\xi}, \cdots, \nu_{\xi+n-1}\right\}$. Then $A_{1}$ and $A_{2}$ are $C_{\nu_{0}}(\alpha)$-equivalent by virtue of condition (Ek) for some $k=1, \cdots$, 5. In each case a contradiction ensues.

$(k=1)$ Then $b\left(\nu_{0}\right)<b\left(\nu_{1}\right)<\cdots<b\left(\nu_{n}\right)$; and, hence, if $\xi_{0}<\xi_{1}<\gamma$, then $b\left(\nu_{\zeta_{0}}\right)<b\left(\nu_{\zeta_{1}}\right)$. But then $\left\{b\left(\nu_{\xi}\right): \xi<\gamma\right\}$ is D-homogeneous and has length $\gamma$.

$(k=2) b\left(\nu_{0}\right)=b\left(\nu_{1}\right)<\cdots<b\left(\nu_{n}\right)$ and $b\left(\nu_{0}\right)=b\left(\nu_{2}\right)<\cdots<b\left(\nu_{n+1}\right)$ which is impossible.

$(k=3) b\left(\nu_{0}\right) \leq b\left(\nu_{1}\right)=\cdots=b\left(\nu_{n+1}\right)$. Hence, if $0<\xi<\gamma$, then $b\left(\nu_{\xi}\right)=b\left(\nu_{1}\right)$. Thus $X$ is $C\left(g\left(b\left(\nu_{1}\right)+1\right)\right)$-homogeneous. 
$(k=4)$ In this case $n \geq 3$. There is a nonzero $i \leq n-2$ such that either $\nu_{i}=$ $\nu_{i+1}<\nu_{i+2}$ or $\nu_{i}<\nu_{i+1}=\nu_{i+2}$; but then $\nu_{i+1}=\nu_{i+2}<\nu_{i+3}$ or $\nu_{i+1}$ $<\nu_{i+2}=\nu_{i+3}$ respectively, a contradiction.

$(k=5) \nu_{1} \leq \nu_{0}$, which is impossible.

Thus the lemma is proved.

Proof. of 4.2. It follows from Lemma 4.6 that $S_{2}(0,2,5)$. By repeated applications of Lemmas 4.5 and 4.6 we get that if $n \geq 1$, then $S_{1}(n-1, n+2, n+5)$. Thus, every inaccessible in $\mathbf{P}(n+2, n+5)$, for $n \geq 1$, is already $n$-inaccessible. But by Corollary 2.3, $\mathrm{P}(n+2, n+5)$ contains only inaccessibles.

4.7. Lemma. $S_{2}(0,1, \omega)$.

Proof. Let $\kappa$ be inaccessible and let $f: \kappa \rightarrow \kappa$ be 0 -normal; hence, by Proposition 4.4, $\kappa$ is not 1 -inaccessible. We show by induction on ordinals that for each $\alpha<\kappa$ there exists an $f$-partition system $C(\alpha)$ of $\alpha$ such that every $C(\alpha)$-homogeneous set is finite. Let $\alpha<\kappa$ and assume that for each $\beta<\alpha$ such a $C(\beta)$ exists; we prove such a $C(\alpha)$ exists.

Case 1. $\alpha \leq \omega$. This case is trivial since $f(\nu) \geq \omega \geq a$ whenever $\nu<\kappa$.

Case 2. $\alpha>\omega$ and $\alpha \notin f[\kappa]$. Then there is $\beta$ such that $\beta<\alpha<f(\beta)$. Let $C(\alpha)$ be the partition system of $\alpha$ such that whenever $\nu, \nu_{0}, \nu_{1}<\alpha$, then $\nu_{0}$ and $\nu_{1}$ are $C(\alpha)$-equivalent iff one of the following holds:

(i) $\nu<\beta \leq \nu_{0}$ and $\beta \leq \nu_{1}$.

(ii) $\nu<\beta$, and $\nu_{0}$ and $\nu_{1}$ are $C_{\nu}(\beta)$-equivalent.

(iii) $\beta \leq \nu$ and $\nu_{0}=\nu_{1}$.

Evidently, $C(\alpha)$ is an $f$-partition system of $a$.

Suppose that $X$ is $C(\alpha)$-homogeneous. The induction hypothesis and (ii) jointly imply that $X \cap \beta$ is finite. But (iii) implies that $\operatorname{card}(X-\beta) \leq 2$. Hence, $X$ is finite.

Case 3. $\alpha>\omega$ and $\alpha \in f[k]$. Then $\alpha$ is singular. Let $\lambda=c f(\alpha)$, and let $g: \lambda \rightarrow \alpha$ be continuous, strictly increasing and cofinal in $\alpha$. Let $b: \alpha \rightarrow \lambda$ be such that $b(\nu)=\sup \{\beta<\lambda: g(\beta) \leq \nu\}$. For each $\nu<\alpha$, let $H_{\nu}: \alpha \rightarrow f(\nu)$ be such that whenever $\nu<\alpha$ and $b\left(\nu_{0}\right)=b\left(\nu_{1}\right)$, then $\nu_{0}$ and $\nu_{1}$ are $C_{\nu}\left(g\left(b\left(\nu_{0}\right)+1\right)\right)$ equivalent iff $H_{\nu}\left(\nu_{0}\right)=H_{\nu}\left(\nu_{1}\right)$. Now let $C(\alpha)$ be the partition system of $a$ such that whenever $\nu, \nu_{0}, \nu_{1}<\alpha$, then $\nu_{0}$ and $\nu_{1}$ are $C_{\nu}(\alpha)$-equivalent iff one of the following holds:

(i) $\nu<\nu_{0}$ and $\nu<\nu_{1} ; b\left(\nu_{0}\right)$ and $b\left(\nu_{1}\right)$ are $C_{b(\nu)}(\lambda)$-equivalent; and $H_{\nu}\left(\nu_{0}\right)=$ $H_{\nu}\left(\nu_{1}\right)$.

(ii) $\nu_{0} \leq \nu$ and $\nu_{1} \leq \nu$.

Evidently, $C(\alpha)$ is an $f$-partition system of $\alpha$.

Now suppose that $X$ is an infinite $C(\alpha)$-homogeneous set. Then there is an 
infinite $Y \subset X$ such that $b \mid Y$ is either a constant or a one-one function. Suppose $b \mid Y$ is a constant function; if $\nu=\min (Y)$, then $Y$ is $C(g(b(\nu)+1))$-homogeneous. Suppose $b \mid Y$ is one-one; then $b[Y]$ is an infinite $C(\lambda)$-homogeneous set. Either supposition leads to a contradiction; hence, $X$ is finite.

Proof of 4.1. By Lemma 4.7 and repeated applications of Lemmas 4.5 and 4.6 we get that $S_{1}(n, n+2, \omega+n+1)$. Thus, every inaccessible in $\mathbf{P}(n+2, \omega+n+1)$ is already $(n+1)$-inaccessible. But by Corollary 2.3, $\mathbf{P}(n+2, \omega+n+1)$ contains only inaccessibles.

5. Epilogue. In the following table we give the characterization of the classes $\mathbf{P}(n, \alpha)$. To a void technicalities we restrict our attention to countable $\alpha$ and to those uncountable cardinals $\mathbf{P}(n, a)$.

\begin{tabular}{|c|c|c|c|c|c|c|c|c|c|c|c|c|c|}
\hline & 1 & 2 & 3 & 4 & 5 & 6 & $7 \ldots$ & $\omega$ & $\omega+1$ & $\omega+2$ & $\omega+3$ & \multicolumn{2}{|c|}{$\omega+4 \cdots$} \\
\hline 1 & C & $C$ & C & $R$ & $R$ & $R$ & $R \cdots$ & $R$ & $R$ & $R^{*}$ & $R^{*}$ & $R^{*}$ & $\cdots$ \\
\hline 2 & C & C & C & $S$ & $I_{0}$ & $I_{0}$ & $I_{0} \cdots$ & $?$ & $I_{1}$ & $I_{1}$ & $I_{1}$ & $I_{1}$ & $\cdots \cdot$ \\
\hline 3 & $C$ & C & C & C & $S$ & $I_{1}$ & $I_{1} \cdots$ & $?$ & $?$ & $I_{2}$ & $I_{2}$ & $I_{2}$ & $\cdots \cdot$ \\
\hline 4 & C & C & C & C & $C$ & $S$ & $I_{2} \cdots$ & $?$ & $?$ & $?$ & $I_{3}$ & $I_{3}$ & $\cdots \cdot$ \\
\hline 5 & C & C & C & C & $C$ & C & $s \ldots$ & $?$ & $?$ & $?$ & $?$ & $I_{4}$ & $\cdots$ \\
\hline
\end{tabular}

The classes of uncountable cardinals are abbreviated as follows:

$C=$ class of all uncountable cardinals.

$R=$ class of all regular uncountable cardinals.

$S=$ class of all uncountable strong limit cardinals.

$I_{n}=$ class of all uncountable $n$-inaccessible cardinals.

$R^{*}=$ class of all regular cardinals for which $\mu<\kappa$ implies $\mu^{\omega}<\kappa$.

The class of accessible cardinals in $\mathbf{P}(n, \alpha)$ is completely characterized in \$2. The situation for inaccessible cardinals is not complete, although Theorems 3.2 and 4.2 together give a characterization of the class of $n$-inaccessible cardinals.

5.1. Corollary. $\kappa$ is n-inaccessible iff $\kappa \in \mathbf{P}(n+2, n+5)$.

The full strength of Theorem 3.2 is not used in the above characterization. A weakened form of Theorem 3.2, which is just sufficient for this characterization, can be proved inductively using Theorem 2.2 and (only a slight modification of) Lemma 3.3. In this way Lemmas 3.5 and 3.6 are avoided.

In order that every $\mathbf{P}(n, a)$ be completely characterized, either Theorem 3.2 or Theorem 4.1 (or both) must be strengthened. It seems very plausible that all 
the $n$-inaccessible cardinals should behave uniformly, so that either Theorem 3.2 or 4.1 is already the best possible. This leads to the following (mutually contradictory) conjectures.

5.2. Conjecture. If $\kappa$ is $n$-inaccessible, then $\kappa \in \mathrm{P}(n+2, \omega+n)$.

5.3. Conjecture. If $\kappa \in \mathbf{P}(n+2, \omega)$, then $\kappa$ is $(n+1)$-inaccessible.

Either conjecture results in the characterization of each class $\mathbf{P}(n, a)$.

\section{BIBLIOGRAPHY}

1. P. Erdös and R. Rado, A partition calculus in set theory, Bull. Amer. Math. Soc. 62 (1956), 427-489. MR 18, 458.

2. A. Lévy, Axiom schemata of strong infinity in axiomatic set theory, Pacific J. Math. 10 (1960), 223-238. MR 23 \#A1522.

3. F. P. Ramsey, On a problem of formal logic, Proc. London Math. Soc. 30 (1930), 264-286.

4. J. H. Schmerl, On hyperinaccessible-like models, Notices Amer. Math. Soc. 16 (1969), 843. Abstract \#69T-E59.

5. J. H. Schmerl and S. Shelah, On models with orderings, Notices Amer. Math. Soc. 16 (1969), 840. Abstract \#69T-E50.

6. J. H. Schmerl, On $\kappa$-like models for inaccessible $\kappa$, Doctoral Dissertation, University of California, Berkeley, Calif., 1971.

DEPARTMENT OF MATHEMATICS, YALE UNIVERSITY, NEW HAVEN, CONNECTICUT 06520

Current address: Department of Mathematics, University of Connecticut, Storrs, Connecticut 06268 